\title{
Parametric oscillations of the mechanical systems
}

\author{
Branislav Ftorek $^{1, *}$, Pavol Oršanský ${ }^{1}$, Helena Šamajová ${ }^{1}$ \\ ${ }^{1}$ Department of Applied Mathematics, Faculty of Mechanical Engineering, University of Žilina, \\ Univerzitná 1, 01026 Žilina, Slovakia
}

\begin{abstract}
This paper deals with an approximative solution in the analytical form of some second order linear differential equation with time depend parameters. The analytic solution is compared with the numerical solution obtained by Runge-Kutta method and the conditions of the convergence of this analytical method are discussed.
\end{abstract}

Keywords: oscillations, asymptotic solution, Mathieu equation

\section{Introduction}

Time-periodic modulation of system parameters may lead to resonances, instabilities and some pattern formation. The simplest system exhibiting parametric resonances is the harmonic oscillator with a frequency that varies periodically with time. The equation of motion of a simple harmonic oscillator whose frequency is modulated by a time-dependent zero-average perturbation $\xi(t)$ is

$$
\ddot{x}+\omega_{0}^{2}[1+\xi(t)] x=0,
$$

where $x$ is the displacement and $\omega_{0}$ is the natural frequency of the oscillator. This equation of motion can be solved with a friction term. Unlike a forced harmonic oscillator, the equation (1) has a fixed point at $x=0$ regardless of the type of modulation. When the modulation is absent, the fixed point is neutrally stable. That is, near $x=0$ the displacement neither grows without bound nor decays.

However, in the modulation case the fixed point may be unstable for certain temporal modulations. It is interesting that even linear oscillator with a modulated frequency is a "nonlinear problem" because the modulation occurs in a system parameter and is thus multiplicative (see [1, 2]). An example of unstable mode of oscillation system with its phase portrait can be seen in Figure 1.

\footnotetext{
* Corresponding author: branislav.ftorek@,fstroj.uniza.sk

Reviewers: Radim Halama, Jerzy Winczek
} 

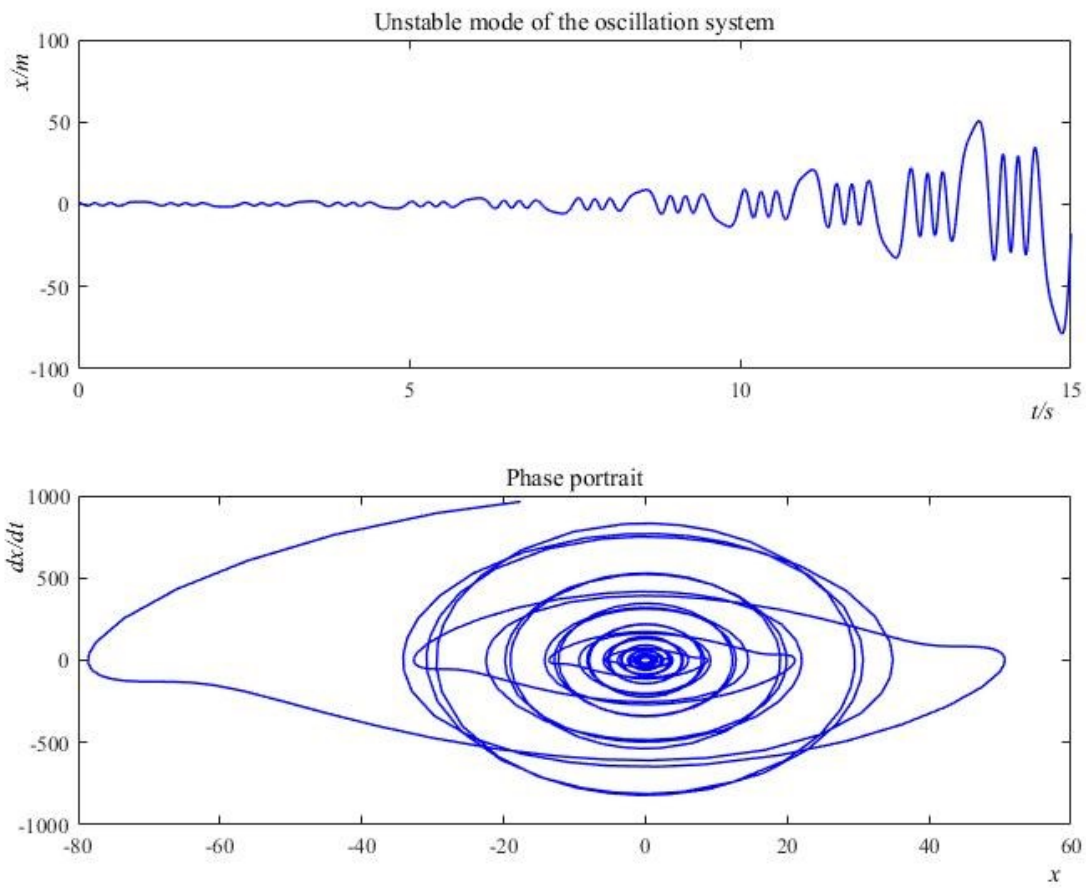

Fig. 1. Unstable mode of oscillation system with its phase portrait:

$$
\ddot{x}+\omega_{0}^{2}[1+\sin B t]=0 \text { where } \omega_{0}=20, B=\frac{\omega_{0}}{4} \text {. }
$$

In most general form, the equation of motion (1) is called Hill's equation

$$
\ddot{x}+p(t) \dot{x}+q(t) x=0,
$$

in which functions $p(t)$ and $q(t)$ are continuous and have a common period $\tau$. If the modulation is specified to be cosinusoidal, the equation (2) is called the Mathieu equation

$$
\ddot{x}+\left[\omega_{0}^{2}-\varepsilon \cos t\right] x=0 .
$$

The solution of the Mathieu equation or the solution of some nonlinear equation may be find by using general asymptotic methods for sufficiently small parameter $\varepsilon, 0<\varepsilon \ll 1$. Many interesting physical phenomena are described by general differential equations with nonlinear or time dependent terms

$$
\ddot{x}+\omega_{0}^{2} x+\varepsilon f(t, x, \dot{x})=0,
$$

where $f(t, x, \dot{x})$ is a real function and $\varepsilon$ is a small parameter. If $\varepsilon=0$ then the solution of the previous equation has a form

$$
x(t)=A \sin \left(\omega_{0} t+\varphi\right),
$$

where the amplitude $A$ and the phase $\varphi$ are constants. We suppose the perturbation solution for $\varepsilon \neq 0$ in the form of an asymptotic series [4]

$$
x(t)=A(t) \sin \left(\omega_{0} t+\varphi(t)\right)+\varepsilon \phi_{1}(t)+\varepsilon^{2} \phi_{2}(t)+\cdots+\varepsilon^{n} \phi_{n}(t)+\cdots,
$$


where $A(t), \varphi(t), \phi_{1}(t), \ldots, \phi_{n}(t), \ldots$ are unknown functions. Another asymptotic method is well known as a WKB-method, or Wentzel-Kramers-Brillouin method (see [5]) for the equation with a large parameter $\lambda$, that is, $\lambda \rightarrow \infty$

$$
\ddot{x}+f(x, \lambda) x=0 .
$$

\section{Asymptotic solution}

Now we consider a second order differential equation with a friction term and with a fast time periodic modulation, that is, the parameter $B$ is sufficiently large

$$
\begin{gathered}
\ddot{x}+2 \gamma \dot{x}+\omega_{0}^{2}[1+\lambda \sin B t] x=0, \\
x(0)=1,
\end{gathered}
$$

where $\lambda>0, \gamma>0, \omega_{0}>0$, are parameters of the oscillation system. Now we will use the basic idea from the previous section and from the paper [6]. If $\lambda=0$ (with regard to the initial condition (9)), the solution has an exact periodic form with damping

$$
x_{0}(t)=e^{-\gamma t} \cos \omega t
$$

where $\omega^{2}=\omega_{0}^{2}-\gamma^{2}>0$. For $\lambda \neq 0$ we assume the solution of (8) as a harmonic modulation of the solution (10)

$$
x(t)=e^{-\gamma t} \cos (\Omega t)[1+\varepsilon \sin B t],
$$

where $\Omega, \varepsilon$ are unknown constant parameters (not functions) and the parameter $\varepsilon$ is sufficiently small, that means, $0<\varepsilon \ll 1$. From (11) we have

$$
\begin{gathered}
=e^{-\gamma t}[-\gamma \cos \Omega t(1+\varepsilon \sin B t)-\Omega \sin \Omega t(1+\varepsilon \sin B t)+\varepsilon B \cos \Omega t \cos \mathrm{B} t], \\
\ddot{x}=e^{-\gamma t}\left[\gamma^{2} \cos \Omega t(1+\varepsilon \sin B t)+2 \gamma \Omega \sin \Omega t(1+\varepsilon \sin B t)\right. \\
-2 \gamma \varepsilon B \cos \Omega t \cos B t-\Omega^{2} \cos \Omega t(1+\varepsilon \sin B t) \\
\left.-2 \Omega \varepsilon B \sin \Omega t \cos \mathrm{B} t-\varepsilon B^{2} \cos \Omega t \sin \mathrm{B} t\right],
\end{gathered}
$$

Substituting the previous relations in (8) and with regard to

$$
\sin ^{2} B t=\frac{1-\cos 2 B t}{2}
$$

we get

$$
\begin{gathered}
e^{-\gamma t}\left\{\operatorname { c o s } \Omega t \left[-\gamma^{2}+\omega_{0}^{2}-\Omega^{2}+\frac{\varepsilon \lambda \omega_{0}^{2}}{2}\right.\right. \\
+\sin \mathrm{B} t\left(\varepsilon \gamma^{2}-\varepsilon \Omega^{2}-\varepsilon B^{2}-2 \varepsilon \gamma^{2}+\omega_{0}^{2} \lambda+\varepsilon \omega_{0}^{2}\right) \\
\left.\left.-\frac{\varepsilon \lambda \omega_{0}^{2}}{2} \cos 2 B t\right]+\sin \Omega t(-2 \varepsilon \Omega B \cos \mathrm{B} t)\right\}=0 .
\end{gathered}
$$
is

It is obvious that the constant term $\left(-\gamma^{2}+\omega_{0}^{2}-\Omega^{2}+\frac{\varepsilon \lambda \omega_{0}^{2}}{2}\right)$ must be equal to zero, that

$$
\Omega=\sqrt{\omega_{0}^{2}-\gamma^{2}+\frac{\varepsilon \lambda \omega_{0}^{2}}{2}}
$$


Now we consider the $\sin B t$ term equals to zero

$$
\varepsilon \gamma^{2}-\varepsilon \Omega^{2}-\varepsilon B^{2}-2 \varepsilon \gamma^{2}+\omega_{0}^{2} \lambda+\varepsilon \omega_{0}^{2}=0 .
$$

According to (16), from the previous condition we have for the second unknown parameter $\varepsilon$ the quadratic equation

$$
\frac{-\lambda \omega_{0}^{2}}{2} \varepsilon^{2}-\varepsilon B^{2}+\omega_{0}^{2} \lambda=0 .
$$

The root of this equation $(\varepsilon>0)$ has the following form

$$
\varepsilon=\frac{-B^{2}}{\lambda \omega_{0}^{2}}+\sqrt{\frac{B^{4}}{\lambda^{2} \omega_{0}^{4}}+2}
$$

The relation (19) represents a condition of applicability of the main approximative method. It is desirable that the parameter $\varepsilon$ is sufficiently small, that is, $\varepsilon \rightarrow 0^{+}$if $B \rightarrow \infty$. From (15) we have a residual term

$$
R=e^{-\gamma t}\left[\cos \Omega t\left(-\frac{\varepsilon \lambda \omega_{0}^{2}}{2} \cos 2 B t\right)+\sin \Omega t(-2 \varepsilon \Omega B \cos B t)\right]
$$

and we suppose that the residuum $R$ is sufficiently small $(R \sim 0)$. The validity of this assumption is verified by numerical experiments. We obtained numerical results by the MATLAB environment. The comparison between analytical solutions and numerical solutions for the large scale of parameters are shown in the graphs, where numerical results represent black dot-dashes lines and analytical results the blue line (see Figures 2-5).

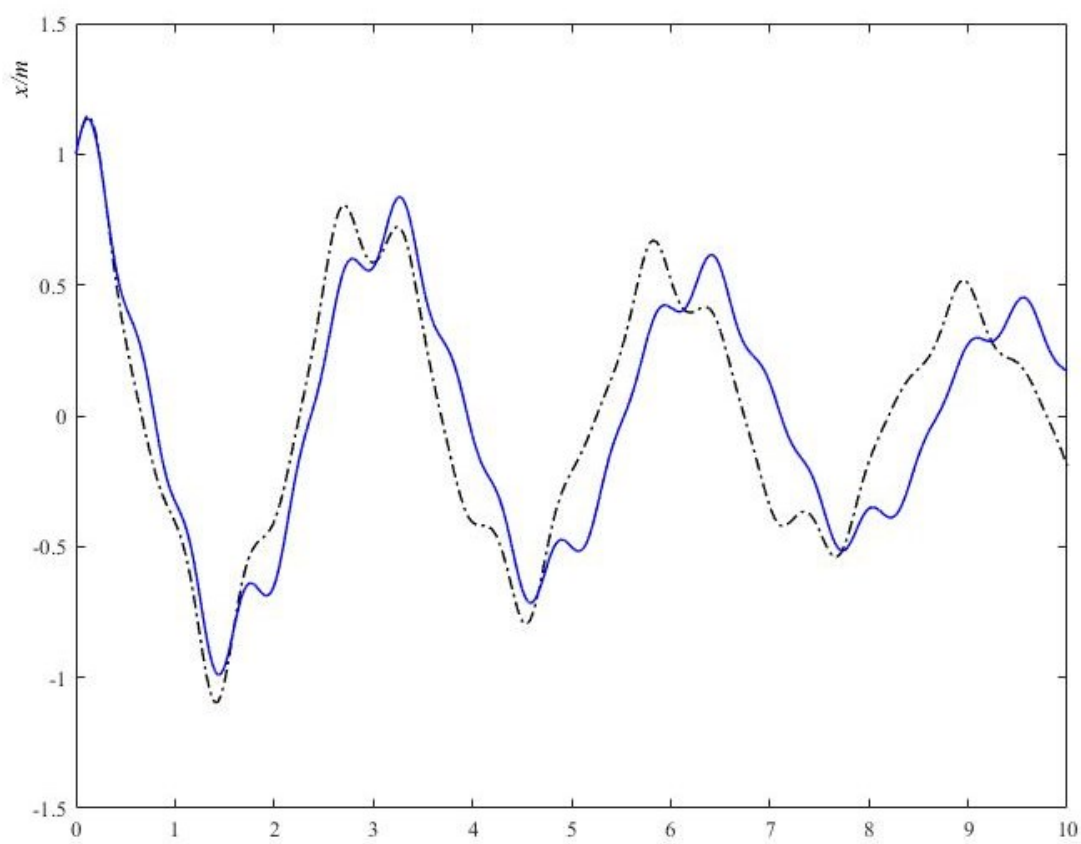

Fig. 2. Comparison between numerical (black) and analytical (blue) solution for parameters:

$$
\gamma=0.1, \omega_{0}=\sqrt{2}, \lambda=10, B=10, \varepsilon=0.196 .
$$




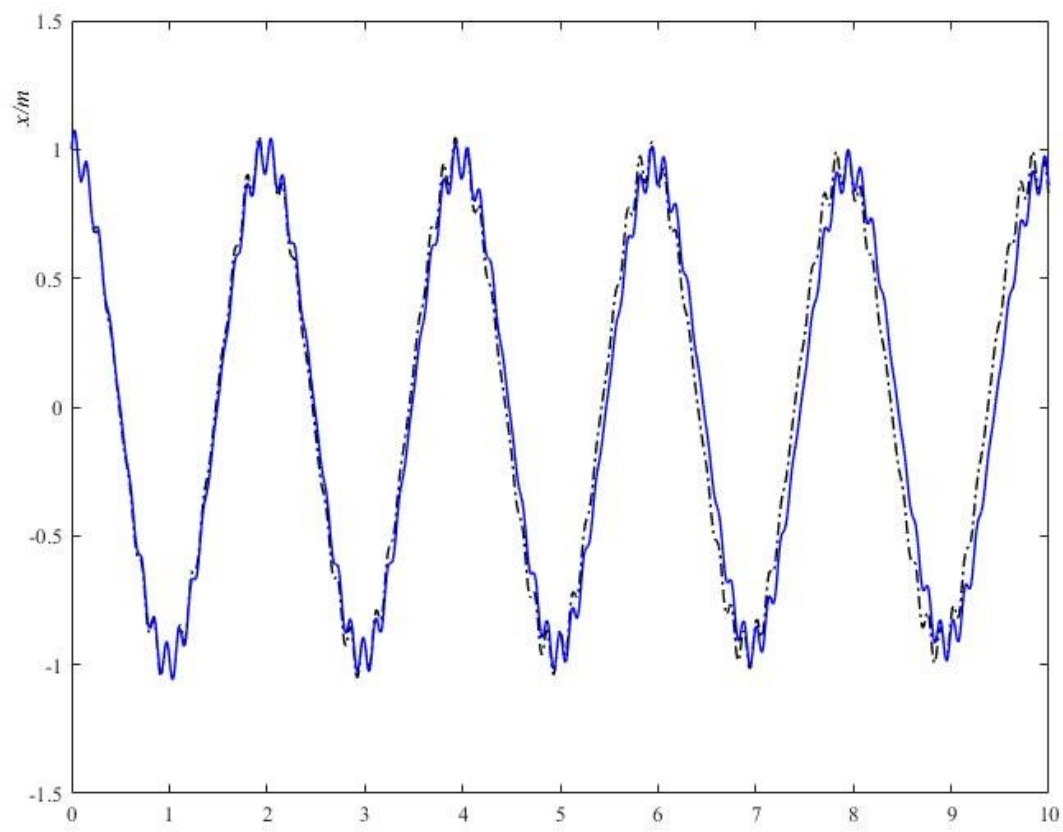

Fig. 3. Comparison between numerical (black) and analytical (blue) solution for parameters:

$$
\gamma=0.1, \omega_{0}=\sqrt{2}, \lambda=100, B=50, \varepsilon=0.08 \text {. }
$$

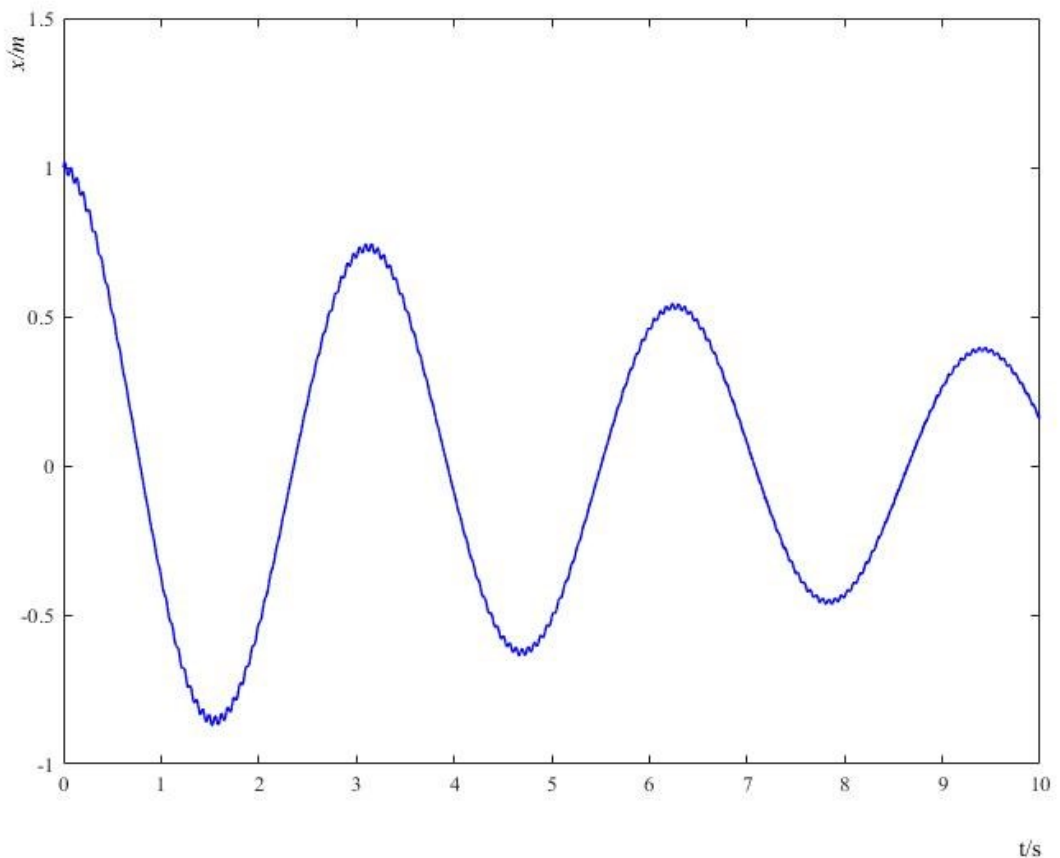

Fig. 4. Comparison between numerical (black) and analytical (blue) solution for parameters:

$$
\gamma=0.1, \omega_{0}=\sqrt{2}, \lambda=100, B=50, \varepsilon=0.02 \text {. }
$$




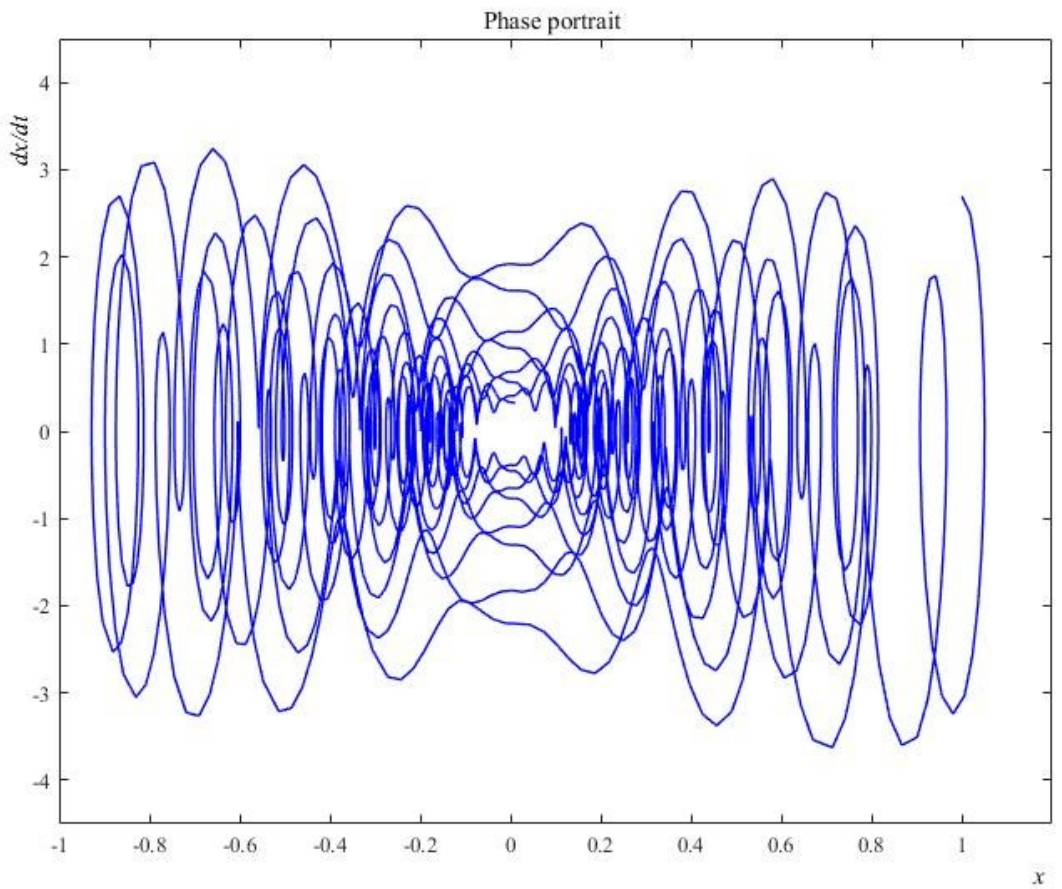

Fig. 5. Stable mode of oscillation system with its phase portrait.

\section{Conclusion}

The equation of motion (8) of a damped harmonic oscillator whose frequency is modulated by a time-dependent zero-average perturbation was investigated. An approximative solution

$$
\begin{gathered}
x(t)=e^{-\gamma t} \cos (\Omega t)[1+\varepsilon \sin B t], \\
\Omega=\sqrt{\omega_{0}^{2}-\gamma^{2}+\frac{\varepsilon \lambda \omega_{0}^{2}}{2}}, \\
\varepsilon=\frac{-B^{2}}{\lambda \omega_{0}^{2}}+\sqrt{\frac{B^{4}}{\lambda^{2} \omega_{0}^{4}}+2,}
\end{gathered}
$$

for a fast time periodic modulation (if $B$ is sufficiently large then the parameter $\varepsilon$ is sufficiently small, that is, $\lim _{B \rightarrow \infty} \varepsilon=0$ ) was obtained. This obtained asymptotic solution was compared with the numerical solution gained by the Runge-Kutta method.

This work was supported by KEGA under the Grant No. 015ŽU-4/2017 Digital technologies for machine building study programs as a part of strategy "Internet of Things".

\section{References}

1. R. Kawai, K. Lindenberg, C. Van den Broeck: Parametrically modulated oscillator dimer: an analytic solution. Physica A 312, 119-140, (2002)

2. S. H. Strogatz: Nonlinear dynamics and chaos. Westview Press, Cambridge, (1994) 
3. J. Zapoměl, V. Dekýš, P. Ferfecki, A. Sapietová, M. Sága, M. Žmindák: Identification of Material Damping of a Carbon Composite Bar and Study of Its Effect on Attenuation of Its Transient Lateral Vibrations. International Journal of Applied Mechanics 7, 1-18, (2015)

4. R. A. Struble: Nonlinear differential equations. McGraw-Hill book company, New York, (1962)

5. J. D. Murray: Asymptotic analysis. Applied mathematical sciences, Springer-Verlag, New-York, (1984)

6. E. Wiszt, E. Wisztová: An approximative solution of some second order differential equation with variable coefficients. Studies of the University of Žilina, Math. Series 21, 45-52 (2007) 